Ecología

\title{
Valoración de metabolitos de testosterona, progesterona y estrógeno en excretas de venado cola blanca (Odocoileus virginianus) como método para determinar el sexo
}

\author{
Measurement of fecal testosterone, progesterone and estrogen metabolites as a method to determine \\ sex in white tailed deer (Odocoileus virginianus) \\ Ermilo Humberto López-Cobá ${ }^{\mathrm{a}, *}$ y Rubén Cornelio Montes-Pérez ${ }^{\mathrm{b}}$ \\ ${ }^{a}$ Departamento de Estudios de Posgrado e Investigación, Instituto Tecnológico de Tizimín, Final Aeropuerto Cupul s/n, 97702, Tizimín, Yucatán, México

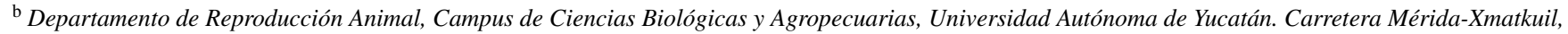 \\ km 15.5, 97300, Mérida, Yucatán, México
}

Recibido el 7 de noviembre de 2014; aceptado el 29 de septiembre de 2015

Disponible en Internet el 13 de febrero de 2016

\begin{abstract}
Resumen
Se obtuvieron excretas de venado cola blanca (Odocoileus virginianus) en cautiverio dentro de unidades de manejo para la conservación de la vida silvestre, ubicadas en Mérida y Ticul, Yucatán, México, para medir las concentraciones de los metabolitos de testosterona, progesterona y estrógeno con la técnica de radioinmunoanálisis y se relacionaron mediante índices. Se obtuvieron concentraciones detectables de los 3 metabolitos y se analizaron los índices mediante la teoría del valor predictivo. Los índices de progesterona /testosterona —ambos a dilución 1:2 y dilución 1:20/1:2, respectivamente - tuvieron la mayor eficiencia (77.5\%) para asignar correctamente el sexo en la época no reproductiva. En la época reproductiva el índice progesterona / testosterona —dilución 1:2/1:20, respectivamente— tuvo la mayor eficiencia (68.75\%). Estas eficiencias son superiores a las obtenidas mediante métodos indirectos para determinar la proporción de sexos en venado cola blanca. El método de determinación del sexo por estimación de los metabolitos de hormonas fecales es factible de ser aplicado en poblaciones silvestres de venado cola blanca. Derechos Reservados (C) 2016 Universidad Nacional Autónoma de México, Instituto de Biología. Este es un artículo de acceso abierto distribuido bajo los términos de la Licencia Creative Commons CC BY-NC-ND 4.0.
\end{abstract}

Palabras clave: Poblaciones; Hormonas; Excretas; Radioinmunoanálisis

\begin{abstract}
We obtained feces of white-tailed deer (Odocoileus virginianus) in captivity from management units for the wildlife conservation, located in Mérida and Ticul, Yucatán, Mexico, in order to measure testosterone, progesterone and estrogen metabolites concentrations using radioinmunoanalysis. We obtained detectable levels of the 3 metabolites, which were analyzed by predictive theory. Indexes progesterone /testosterone -both at 1: 2 dilution and dilution 1:20/1:2, respectively-, had the highest efficiency (77.5\%) to determine sex in non-breeding season properly. During the breeding season the progesterone /testosterone index -dilution 1: 2/1:20, respectively, had the highest efficiency (68.75\%). These efficiencies were higher than those obtained by indirect methods for determining the sex ratio in white-tailed deer. The method of sex determination by estimating fecal hormone metabolites is likely to be applied to wild populations of white-tailed deer.

All Rights Reserved (C) 2016 Universidad Nacional Autónoma de México, Instituto de Biología. This is an open access item distributed under the Creative Commons CC License BY-NC-ND 4.0.
\end{abstract}

Keywords: Populations; Hormones; Feces; Radioinmunoanalysis

\footnotetext{
* Autor para correspondencia.

Correo electrónico: elcoba2003 @ hotmail.com (E.H. López-Cobá).

La revisión por pares es responsabilidad de la Universidad Nacional Autónoma de México.
} 


\section{Introducción}

Determinar la proporción de sexos es primordial en poblaciones sometidas a aprovechamiento realizado de manera selectiva, porque existe una afectación directa a la estructura de edades y de sexos. Este manejo impacta de manera significativa el número de nacimientos en la población, lo que ocasiona al paso del tiempo un desbalance tanto en el tamaño de la población como en la proporción de sexos (Coltman, 2008; Milner, Nilsen y Andreassen, 2007).

El censo o estimación de la proporción de sexos de las poblaciones de venados en condiciones de semicautiverio o vida libre se realiza usualmente mediante el avistamiento directo (Mandujano y Gallina, 1993). En el trópico este método se torna complicado debido a la baja visibilidad de los ejemplares en los diferentes tipos de vegetación —bosques caducifolios, subcaducifolios, subperennifolios o perennifolios y vegetación secundaria- y a los hábitos furtivos de esta especie (Weber, García-Marmolejo y Reyna-Hurtado, 2006). Entre los métodos que se han establecido para determinar el sexo en venados, además de los registros visuales (Mandujano y Gallina, 1996; Sánchez-Rojas, Gallina y Equihua, 2004), se encuentran los indirectos como la morfometría de pellets (CamargoSanabria y Mandujano, 2009; Sánchez-Rojas, Aguilar-Miguel y Hernández-Cid, 2009; Sánchez-Rojas et al., 2004), la espectroscopía reflectante de infrarrojo cercano (Tolleson, Randel, Stuth y Neuendorff, 2005) y el análisis de ADN (Lindsay y Belant, 2008). Los métodos indirectos aplicados a las excretas del venado cola blanca presentan como inconveniente el tener una eficiencia en la correcta determinación del sexo cercana al 40\% (Camargo-Sanabria, 2008), del 0\% en machos y del $100 \%$ en hembras gestantes, únicamente en la época reproductiva (Tolleson et al., 2005); por su parte, la determinación del sexo por medio del análisis de ADN requiere de la colecta de muestras específicas de tejido (Lindsay y Belant, 2008), lo cual es poco viable en venados de vida libre en las regiones tropicales.

El método de determinación del sexo a través de hormonas esteroides sexuales se ha utilizado en algunos mamíferos silvestres con eficiencias cercanas al 100\% (Barja, Silván, Illera, Rosellini y Piñeiro, 2006; Lanyon, Smith y Carrick, 2005; Soto, Salamé-Méndez, Ramírez-Pulido, Yáñez y Armella, 2004). Estos estudios demostraron que los andrógenos son las principales hormonas indicadoras de los machos y los estrógenos lo son de las hembras, y su producción relativa puede usarse como un indicador del sexo. Este método ha permitido determinar correctamente el sexo en individuos tanto en cautiverio como en vida libre (Barja et al., 2006; Soto et al., 2004).

En cautiverio, el método tradicional de medición de hormonas esteroides de origen gonadal es practicada en muestras sanguíneas, tal como sucede en la monitorización reproductiva de ganado bovino u ovino (De Lucas, Zarco y Vásquez, 2008; Herzog et al., 2010). Sin embargo, la aplicación de este método es complicada en venados cola blanca (Odocoileus virginia$n u s)$, debido a su temperamento nervioso, desencadenando un aumento de estrés y elevando los niveles de progesterona sanguínea de origen adrenal (Plotka, Seal, Verme y Ozoga, 1983), así como respuestas conductuales que ponen en riesgo el bienestar del ejemplar o que pueden desencadenar el síndrome de miopatía por captura (Beringer, Hansen, Wilding, Fischer y Sheriff, 1996; Haulton, Porter y Rudolph, 2001). Por estos factores, actualmente se utilizan métodos no invasivos como la medición de progestinas, andrógenos y estrógenos en excretas, los cuales deben ser validados para cada especie antes de su aplicación (Palme, 2005). Por ello, los objetivos de este trabajo son establecer un protocolo para la estimación de metabolitos de hormonas esteroides sexuales mediante radioinmunoanálisis (RIA, por sus siglas en inglés) en excretas de venado cola blanca mantenidos en cautiverio e identificar los índices de metabolitos de hormonas esteroides sexuales que muestren mayor eficiencia para la correcta determinación del sexo bajo el contexto de la teoría del valor predictivo.

\section{Materiales y métodos}

Las muestras de excretas de venado cola blanca fueron recolectadas de 3 unidades de manejo para la conservación de la vida silvestre (UMA) de tipo intensivo, ubicadas en las ciudades de Mérida y Ticul en Yucatán, México. La UMA «Xmatkuil» se sitúa en las coordenadas $20^{\circ} 51^{\prime} 48.9^{\prime \prime} \mathrm{O}, 89^{\circ} 36^{\prime} 50.2^{\prime \prime} \mathrm{N}$, la UMA «El Centenario» en $20^{\circ} 58^{\prime \prime} 09.8^{\prime \prime} \mathrm{O}, 89^{\circ} 38^{\prime \prime} 24.7^{\prime \prime} \mathrm{N}$ y la UMA «San Fernando» en $20^{\circ} 23^{\prime} 41.2^{\prime \prime} \mathrm{O}, 89^{\circ} 31^{\prime} 28.2^{\prime \prime} \mathrm{N}$.

Se recolectaron un total de 160 excretas de venados adultos en cautiverio, 80 de machos y 80 de hembras, en época reproductiva (ER) y no reproductiva (ENR), muestras para cada sexo, para cada época. Cada muestra fue tomada del suelo inmediatamente después de que el animal defecó, registrando: fecha, sexo, época y sitio de colecta. Se recolectaron los pellets superiores para evitar las impurezas propias del terreno. Las excretas obtenidas se refrigeraron para su traslado y posteriormente se congelaron $\mathrm{a}-20^{\circ} \mathrm{C}$, hasta su procesamiento (Barja et al., 2006).

La extracción de esteroides se realizó mediante la técnica descrita por Latner, Rajamahendran y Sivakumaran (1994) modificada por Montes, Cervera y Victoria (1999). En breve, las muestras fueron secadas en un horno a $60^{\circ} \mathrm{C}$ por $48 \mathrm{~h}$, posteriormente se molieron lo más finamente posible utilizando una licuadora convencional (Moulinex mod. 4090). Se colocaron $0.5 \mathrm{~g}$ de cada muestra en un tubo de ensayo y se agregó $1 \mathrm{ml}$ de agua destilada; una vez hidratada la muestra se agregaron $3 \mathrm{ml}$ de éter dietílico (Jalmek \#E6525-15) y se agitó en un vortex por $2 \mathrm{~min}$. Posteriormente, se congeló la muestra en baño de nitrógeno líquido y se decantó la fase orgánica a un recipiente. Este proceso se repitió 2 veces más por muestra. Una vez obtenida la fase orgánica se dejó evaporar el solvente, reconstituyéndolo en $10 \mathrm{ml}$ de metanol al 99\%, se agitó en vortex por $1 \mathrm{~min}$, y se refrigeró hasta el momento de analizar. Se probaron 2 diluciones del extracto restituido con metanol, para establecer una dilución adecuada para procesar los extractos. Se adaptó la técnica de RIA, a partir de 2 componentes primarios - anticuerpo y trazador que es la hormona radiactivade un estuche comercial para progesterona (Diagnostics Systems Laboratories cat. 3900), testosterona (Diagnostics Systems Laboratories cat. 4000) y estradiol (Coat-A-Count cat. TKE2). Los estándares fueron preparados con progesterona (P4) (Sigma 
Tabla 1

Características que se evalúan con la teoría del valor predictivo.

\begin{tabular}{lll}
\hline & $\begin{array}{l}\text { Sexo conocido } \\
\text { hembra }\end{array}$ & $\begin{array}{l}\text { Sexo conocido } \\
\text { macho }\end{array}$ \\
\hline Hembra mediante RIA & $\begin{array}{l}\text { Verdadera } \\
\text { hembra }(\mathrm{VH})\end{array}$ & $\begin{array}{l}\text { Falsa hembra } \\
(\mathrm{FH})\end{array}$ \\
Macho mediante RIA & $\begin{array}{l}\text { Falso macho } \\
\text { (FM) }\end{array}$ & $\begin{array}{l}\text { Verdadero } \\
\text { macho }(\mathrm{VM})\end{array}$ \\
\hline
\end{tabular}

cat. P0139-25G), testosterona (T) (Fluka cat. 86500) y estradiol (E2) (Sigma cat. E1024-25G) disueltos en metanol y PBS (Montes, 1994; Montes et al., 1999), y se establecieron las diluciones a las que se trabajaron los extractos de metabolitos (1:2,1:20 para P4 y T, y de 1:2 y 1:10 para E2).

Los valores de las concentraciones de los metabolitos para cada concentración y época fueron comparados mediante análisis de varianzas. La validación del RIA se efectuó mediante los criterios de sensibilidad y precisión (según IAEA, 1984). A 3 muestras fecales en 3 repeticiones se les evaluaron los valores de sensibilidad, la cual se definió como la menor cantidad de metabolito hormonal que puede ser detectado con certeza estadística, calculada para el 95\% de confiabilidad en el 0 de la curva estándar y precisión, y que se midió mediante la variación intraensayo e interensayo de muestras con niveles bajos, medios y altos de metabolitos hormonales. La exactitud y especificidad no se evaluaron, debido a que los anticuerpos utilizados fueron validados previamente por los fabricantes de los kits, para enlazar hormonas sanguíneas y no para metabolitos, que corresponden a las formas oxo o diol (Hamilton et al., 2000; Rabiee, Macmillan y Schwarzenberg, 2001).

De los resultados de las estimaciones de las concentraciones de los metabolitos fecales de P4, T y E2, se obtuvieron los índices mediante el cálculo de las relaciones E2/T, T/E2, P4/T, T/P4 y la suma de los 3 metabolitos ( $\mathrm{P} 4+\mathrm{T}+\mathrm{E} 2)$ para cada sexo (Soto et al., 2004), en combinaciones de acuerdo con las diluciones realizadas para cada metabolito. Se lograron valores de corte para asignar el sexo por cada metabolito e índice. Se evaluó la eficiencia del diagnóstico remoto del sexo conforme a la teoría de valor predictivo, obteniéndose los valores de eficiencia total y el valor predictivo para asignar sexo tanto a hembras como a machos (Galen, 1979). La teoría de valor predictivo se basa en la tabla de contingencia (tabla 1), la cual se ajustó a lo evaluado:

La fórmula de eficiencia se obtiene de la siguiente manera:

$$
\text { Eficiencia }=\frac{\mathrm{VH}+\mathrm{VM}}{\mathrm{VH}+\mathrm{FH}+\mathrm{VM}+\mathrm{FM}}
$$

El valor predictivo para hembras (VPH) es:

$$
\mathrm{VPH}=\frac{\mathrm{VH}}{\mathrm{VH}+\mathrm{FH}} \times 100
$$

y para machos (VPM):

$$
\mathrm{VPM}=\frac{\mathrm{VM}}{\mathrm{VM}+\mathrm{FM}} \times 100
$$

donde: VH es el número de muestras cuyo sexo conocido es hembra y mediante la valoración hormonal resultó ser hembra. VM es el número de muestras cuyo sexo conocido es macho y mediante la valoración hormonal de RIA resultó ser macho. FH es el número de muestras cuyo sexo conocido es macho y mediante la valoración hormonal resultó hembra. FM es el número de muestras cuyo sexo conocido es hembra y mediante la valoración hormonal con RIA resultó macho.

\section{Resultados}

Todas las muestras fecales tuvieron valores detectables de los metabolitos de progestágenos, andrógenos y estrógenos en las 2 diluciones utilizadas, 1:2 y 1:20 para andrógenos y progestágenos, y 1:2 y 1:10 para estrógenos. Para los metabolitos de progestágenos la sensibilidad fue de $0.29 \mathrm{ng} / \mathrm{ml}$, la precisión del ensayo tuvo un coeficiente de variación interensayo del $9 \%$ e intraensayo del 5.35\%. Para los metabolitos de andrógenos la sensibilidad fue de $0.54 \mathrm{ng} / \mathrm{ml}$ y la variación interensayo fue del $42 \%$ e intraensayo del $4 \%$. Para los metabolitos de estrógenos la sensibilidad fue de $0.024 \mathrm{ng} / \mathrm{ml}$ y la variación interensayo fue del $8 \%$ e intraensayo del $31 \%$.

En la ENR se encontraron diferencias estadísticas entre los valores de los metabolitos de P4 para la dilución 1:2, no así

Tabla 2

\begin{tabular}{|c|c|c|c|c|}
\hline Metabolito & Dilución & Sexo & Época no reproductiva ng/g (DE) & Época reproductiva ng/g (DE) \\
\hline \multirow{2}{*}{ P4 } & & M & $8,066.89( \pm 6,436.82) b$ & $10,797.13( \pm 7,137.36) b$ \\
\hline & & M & $14,211.28( \pm 38,805.45)$ a & $9,211.09( \pm 10,412.50) b$ \\
\hline \multirow[t]{3}{*}{$\mathrm{T}$} & $1: 2$ & $\mathrm{H}$ & $5,333.94( \pm 3,732.08) \mathrm{a}$ & $12,436.87( \pm 13,711.35) \mathrm{a}$ \\
\hline & & M & $7,521.69( \pm 8,049.66) \mathrm{a}$ & $15,151.44( \pm 12,282.47) \mathrm{a}$ \\
\hline & $1: 20$ & $\mathrm{H}$ & $2,879.01( \pm 3,826.50) \mathrm{a}$ & 6,796.71 (土9,196.38) а \\
\hline \multirow{3}{*}{ E2 } & & M & $88.64( \pm 83.94) b$ & $329.67( \pm 1,010.82) \mathrm{a}$ \\
\hline & $1: 10$ & $\mathrm{H}$ & $487.61( \pm 777.68) \mathrm{a}$ & $113.52( \pm 113.74) \mathrm{a}$ \\
\hline & & M & $132.45( \pm 397.79) b$ & $398.22( \pm 1,101.66) \mathrm{a}$ \\
\hline
\end{tabular}

Valores promedio de los metabolitos por época de las muestras de sexo conocido de venado cola blanca por medio de radioinmunoanálisis a diferentes diluciones. Los valores de las concentraciones de los metabolitos para cada concentración y época fueron comparados mediante pruebas de análisis de varianzas.

E2: metabolitos de estradiol; H: hembra; M: macho; P4: metabolitos de progesterona; T: metabolitos de testosterona. Letras diferentes en la misma columna de cada pareja de sexos significa diferencias significativas entre medianas $(p<0.05)$ 
Tabla 3

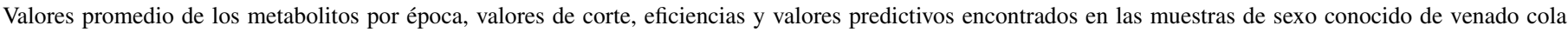
blanca por medio de radioinmunoanálisis a diferentes diluciones.

\begin{tabular}{|c|c|c|c|c|c|c|c|c|c|}
\hline \multirow[b]{2}{*}{ Metabolito } & \multirow[b]{2}{*}{ Dilución } & \multicolumn{4}{|c|}{ Época no reproductiva } & \multicolumn{4}{|c|}{ Época reproductiva } \\
\hline & & $\begin{array}{l}\text { Valor de } \\
\text { corte ng/g }\end{array}$ & Eficiencia \% & $\begin{array}{l}\text { VP\% } \\
\text { hembras }\end{array}$ & $\begin{array}{l}\text { VP\% } \\
\text { machos }\end{array}$ & $\begin{array}{l}\text { Valor de } \\
\text { corte ng/g }\end{array}$ & Eficiencia \% & $\begin{array}{l}\text { VP\% } \\
\text { hembras }\end{array}$ & $\begin{array}{l}\text { VP\% } \\
\text { machos }\end{array}$ \\
\hline \multirow{2}{*}{$\mathrm{P} 4$} & $1: 2$ & 1,100 & 72.5 & 69.57 & 76.47 & 4,800 & 78.75 & 79.49 & 78.05 \\
\hline & $1: 20$ & 9,000 & 73.75 & 70.21 & 78.79 & 14,134 & 65 & 71.43 & 61.54 \\
\hline \multirow{2}{*}{$\mathrm{T}$} & $1: 2$ & 4,321 & 56.25 & 55.56 & 57.14 & 5,749 & 62.5 & 66.67 & 60 \\
\hline & $1: 20$ & 4,800 & 52.5 & 51.61 & 55.56 & 1,624 & 68.75 & 80 & 63.64 \\
\hline \multirow{2}{*}{$\mathrm{E} 2$} & $1: 2$ & 95 & 77.5 & 75 & 80.56 & 108 & 47.5 & 47.62 & 47.37 \\
\hline & $1: 10$ & 151 & 70 & 78.57 & 63.38 & 57 & 53.75 & 56 & 52.73 \\
\hline
\end{tabular}

E2: metabolitos de estradiol; P4: metabolitos de progesterona; T: metabolitos de testosterona; VP: valores predictivos.

para la dilución 1:20; para los metabolitos T2 no se hallaron diferencias en las diluciones evaluadas, y para los metabolitos de E2 se encontraron diferencias significativas en ambas diluciones (tabla 2). Para la ER se encontraron diferencias estadísticas entre los valores de los metabolitos de P4 para ambas diluciones; para el metabolito T2 se hallaron diferencias en la dilución 1:20 y en las diluciones de E2 no (tabla 2). De los valores de los metabolitos hormonales obtenidos en RIA mediante el modelo predictivo, se encontró mayor eficiencia para P4 en la ER en dilución 1:2, seguida de E2 de la ENR en dilución 1:2. La más baja eficiencia para asignar el sexo la tuvo el metabolito hormonal de E2 a dilución 1:2 de la ER (tabla 3). Se obtuvieron 64 índices producto de las diversas combinaciones de metabolitos y diluciones. Los que mejor eficiencia mostraron para la ENR fueron P4/T ambos a dilución 1:2, así como P4/T en diluciones de 1:20 y 1:2 (tabla 4). Para la ER el índice con mayor eficiencia fue P4/T con dilución 1:2 y 1:20 (tabla 5).

Tabla 4

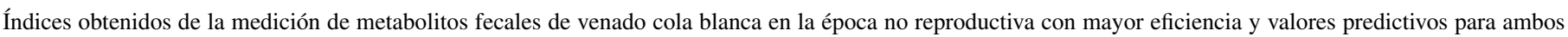
sexos (entre paréntesis la dilución a la que se trabajaron las muestras).

\begin{tabular}{|c|c|c|c|c|}
\hline Índice & Valor de corte & Eficiencia & VP\% hembras & VP\% machos \\
\hline $\mathrm{P} 4(1: 20) / \mathrm{T}(1: 2)$ & 3.8 & 77.5 & 80.56 & 75 \\
\hline $\mathrm{P} 4(1: 2) / \mathrm{T}(1: 2)$ & 2.9 & 77.5 & 76.19 & 78.95 \\
\hline$\Sigma \mathrm{P} 4(1: 20), \mathrm{T}(1: 2), \mathrm{E} 2(1: 10)$ & 18,000 & 72.5 & 72.5 & 72.5 \\
\hline$\Sigma \mathrm{P} 4(1: 2), \mathrm{T}(1: 20), \mathrm{E} 2(1: 10)$ & 12,500 & 71.25 & 68.89 & 74.29 \\
\hline$\Sigma \mathrm{P} 4(1: 2), \mathrm{T}(1: 2), \mathrm{E} 2(1: 10)$ & 16,823 & 71.25 & 69.77 & 72.97 \\
\hline$\Sigma \mathrm{P} 4(1: 2), \mathrm{T}(1: 20), \mathrm{E} 2(1: 2)$ & 12,500 & 71.25 & 68.89 & 74.29 \\
\hline $\mathrm{E} 2(1: 2) / \mathrm{T}(1: 20)$ & 0.22 & 70 & 83.33 & 64.29 \\
\hline $\mathrm{E} 2(1: 2) / \mathrm{T}(1: 2)$ & 0.014 & 70 & 64.29 & 83.33 \\
\hline $\mathrm{T}(1: 2) / \mathrm{E} 2(1: 2)$ & 75 & 70 & 64.29 & 83.33 \\
\hline$\Sigma \mathrm{P} 4(1: 2), \mathrm{T}(1: 2), \mathrm{E} 2(1: 2)$ & 12,312 & 67.5 & 62.96 & 76.92 \\
\hline $\mathrm{T}(1: 20) / \mathrm{E} 2(1: 2)$ & 17.5 & 65 & 61.54 & 71.43 \\
\hline $\mathrm{E} 2(1: 10) / \mathrm{T}(1: 2)$ & 0.004 & 65 & 62 & 70 \\
\hline$\Sigma \mathrm{P} 4(1: 20), \mathrm{T}(1: 2), \mathrm{E} 2(1: 2)$ & 24,271 & 65 & 71.43 & 61.54 \\
\hline$\Sigma \mathrm{P} 4(1: 20), \mathrm{T}(1: 20), \mathrm{E} 2(1: 10)$ & 21,112 & 65 & 71.43 & 61.54 \\
\hline ¿P4(1:20),T(1:20),E2(1:2) & 21,126 & 65 & 71.43 & 61.54 \\
\hline $\mathrm{P} 4(1: 20) / \mathrm{T}(1: 20)$ & 13 & 63.75 & 68.97 & 60.78 \\
\hline E2(1:10)/T(1:20) & 0.004 & 62.5 & 60 & 66.67 \\
\hline $\mathrm{P} 4(1: 20) / \mathrm{E} 2(1: 10)$ & 22 & 61.25 & 59.18 & 64.52 \\
\hline $\mathrm{P} 4(1: 2) / \mathrm{T}(1: 20)$ & 9 & 58.75 & 60.61 & 57.45 \\
\hline $\mathrm{E} 2(1: 2) / \mathrm{P} 4(1: 20)$ & 0.007 & 58.75 & 57.45 & 60.61 \\
\hline $\mathrm{P} 4(1: 2) / \mathrm{E} 2(1: 10)$ & 35 & 58.75 & 57.45 & 60.61 \\
\hline $\mathrm{P} 4(1: 20) / \mathrm{E} 2(1: 2)$ & 175 & 53.75 & 52.73 & 56 \\
\hline $\mathrm{T}(1: 2) / \mathrm{E} 2(1: 10)$ & 2,800 & 52.5 & 75 & 51.32 \\
\hline $\mathrm{E} 2(1: 10) / \mathrm{P} 4(1: 20)$ & 0.021 & 52.5 & 55.56 & 51.61 \\
\hline $\mathrm{E}(1: 2) / \mathrm{P} 4(1: 2)$ & 0.013 & 52.5 & 52.38 & 52.63 \\
\hline $\mathrm{E} 2(1: 10) / \mathrm{P} 4(1: 2)$ & 0.06 & 51.25 & 50.72 & 54.55 \\
\hline $\mathrm{T}(1: 20) / \mathrm{E} 2(1: 10)$ & 2.2 & 51.25 & 51.22 & 51.28 \\
\hline $\mathrm{P} 4(1: 2) / \mathrm{E} 2(1: 2)$ & 92 & 48.75 & 48.78 & 48.72 \\
\hline $\mathrm{T}(1: 20) / \mathrm{P} 4(1: 20)$ & 0.06 & 45 & 45.65 & 44.12 \\
\hline $\mathrm{T}(1: 20) / \mathrm{P} 4(1: 2)$ & 0.09 & 45 & 45.45 & 44.44 \\
\hline $\mathrm{T}(1: 2) / \mathrm{P} 4(1: 2)$ & 0.15 & 43.75 & 46.58 & 14.29 \\
\hline $\mathrm{T}(1: 2) / \mathrm{P} 4(1: 20)$ & 0.9 & 33.75 & 6.67 & 40 \\
\hline
\end{tabular}

E2: metabolitos de estradiol; P4: metabolitos de progesterona; T: metabolitos de testosterona; VP: valores predictivos. 
Tabla 5

Índices obtenidos de la medición de metabolitos fecales de venado cola blanca en la época reproductiva con mayor eficiencia y valores predictivos para ambos sexos (entre paréntesis la dilución a la que se trabajaron las muestras).

\begin{tabular}{|c|c|c|c|c|}
\hline Índice & Valor de corte & Eficiencia & VP\% hembras & VP\% machos \\
\hline $\mathrm{P} 4(1: 20) / \mathrm{T}(1: 20)$ & 12.5 & 66.25 & 88.24 & 60.32 \\
\hline $\mathrm{T}(1: 20) / \mathrm{E} 2(1: 2)$ & 19 & 65 & 71.43 & 61.54 \\
\hline E2(1:10)/P4(1:2) & 0.007 & 60 & 62.5 & 58.33 \\
\hline $\mathrm{T}(1: 20) / \mathrm{E} 2(1: 10)$ & 66 & 60 & 58.33 & 62.5 \\
\hline $\mathrm{P} 4(1: 20) / \mathrm{E} 2(1: 10)$ & 38 & 58.75 & 57.45 & 60.61 \\
\hline $\mathrm{E} 2(1: 2) / \mathrm{T}(1: 2)$ & 0.01 & 57.5 & 56.52 & 58.82 \\
\hline $\mathrm{P} 4(1: 20) / \mathrm{T}(1: 2)$ & 1 & 57.5 & 58.82 & 56.52 \\
\hline $\mathrm{T}(1: 2) / \mathrm{E} 2(1: 10)$ & 77.5 & 57.5 & 56.52 & 58.82 \\
\hline $\mathrm{E} 2(1: 10) / \mathrm{P} 4(1: 20)$ & 0.016 & 56.25 & 55.56 & 57.14 \\
\hline $\mathrm{P} 4(1: 2) / \mathrm{T}(1: 2)$ & 1.3 & 56.25 & 57.14 & 55.56 \\
\hline $\mathrm{E} 2(1: 10) / \mathrm{T}(1: 2)$ & 0.014 & 55 & 55.56 & 54.55 \\
\hline$\Sigma \mathrm{P} 4(1: 2), \mathrm{T}(1: 20), \mathrm{E} 2(1: 10)$ & 21,192 & 52.5 & 52.38 & 52.63 \\
\hline$\Sigma \mathrm{P} 4(1: 20), \mathrm{T}(1: 20), \mathrm{E} 2(1: 10)$ & 20,223 & 52.5 & 52.63 & 52.38 \\
\hline$\Sigma \mathrm{P} 4(1: 20), \mathrm{T}(1: 20), \mathrm{E} 2(1: 2)$ & 20,783 & 52.5 & 52.63 & 52.38 \\
\hline $\mathrm{E}(1: 2) / \mathrm{P} 4(1: 2)$ & 0.013 & 51.25 & 51.22 & 51.28 \\
\hline$\Sigma \mathrm{P} 4(1: 2), \mathrm{T}(1: 2), \mathrm{E} 2(1: 10)$ & 24,129 & 51.25 & 51.22 & 51.28 \\
\hline$\Sigma \mathrm{P} 4(1: 2), \mathrm{T}(1: 2), \mathrm{E} 2(1: 2)$ & 24,119 & 51.25 & 51.22 & 51.28 \\
\hline$\Sigma \mathrm{P} 4(1: 2), \mathrm{T}(1: 20), \mathrm{E} 2(1: 2)$ & 19,405 & 51.25 & 51.28 & 51.22 \\
\hline $\mathrm{E} 2(1: 10) / \mathrm{T}(1: 20)$ & 0.014 & 46.3 & 45.95 & 46.61 \\
\hline $\mathrm{T}(1: 2) / \mathrm{P} 4(1: 20)$ & 1 & 43.75 & 44.44 & 42.86 \\
\hline $\mathrm{T}(1: 2) / \mathrm{E} 2(1: 2)$ & 104 & 42.5 & 41.18 & 43.48 \\
\hline $\mathrm{T}(1: 20) / \mathrm{P} 4(1: 20)$ & 1 & 42.22 & 47.37 & 38.46 \\
\hline $\mathrm{T}(1: 2) / \mathrm{P} 4(1: 2)$ & 0.6 & 41.25 & 42.55 & 39.39 \\
\hline
\end{tabular}

E2: metabolitos de estradiol; P4: metabolitos de progesterona; T: metabolitos de testosterona; VP: valores predictivos.

\section{Discusión}

La sensibilidad de nuestras pruebas para los distintos metabolitos hormonales se encuentra dentro de los parámetros establecidos para determinar como válida una prueba inmunológica (Bedolla-Tovar, Ulloa-Aguirre, Landeros-Valpeña y Pérez-Palacios, 1984). En la mayoría de los RIA, los valores de variación intraensayo fueron menores al $10 \%$, excepto en metabolitos de estradiol, en tanto que la variación interensayo presentó algunos valores arriba de lo adecuado, caso de la testosterona (Bedolla-Tovar et al., 1984), y podrían deberse a las pocas repeticiones realizadas $(n=3)$ para cada ensayo, ya que con un solo valor extremo la variación aumenta. Los resultados del metabolito de E2 muestran un comportamiento diferente por sexo y época, indicando que algunas de las hembras a las que se les tomaron las muestras estaban preñadas en la ENR (Plotka, Seal, Schmoller, Karns y Keenlyne, 1977). En los machos, el observar una mayor cantidad de metabolitos de E2 en la ER se explica por la importancia que tiene esta hormona en la diferenciación de las células germinales masculinas, y su incremento está ligado al aumento de la testosterona y la producción de espermatozoides (Méndez-Villalobos, Jaramillo, Aragón, Ayala y Domínguez-Vara, 2009).
En estudios hormonales realizados en lobo mexicano (Canis lupus baileyi) e ibérico (C. lupus signatus), se ha detectado una mayor concentración de $\mathrm{T}$ en machos $\mathrm{y}$, por tanto, la relación T/P4 también es mayor (Barja et al., 2006; Soto et al., 2004). Basándonos en ello, tanto machos como hembras, pudieran ser clasificados por sus valores de metabolitos fecales de P4, $\mathrm{T}$ o E2 de manera aislada, lo cual se pudo corroborar en este trabajo, ya que el metabolito de P4 tuvo una eficiencia más alta que cualquiera de los índices empleados, pero solo para la ER, en tanto que para la ENR se pueden utilizar los niveles hormonales del metabolito de E2. A pesar de estos resultados es más conveniente trabajar con índices, puesto que se basan en la proporción encontrada de los metabolitos más que en los valores absolutos hallados, los cuales podrían variar debido a las metodologías aplicadas para su determinación.

En los trabajos realizados con lobos, los índices exitosos para distinguir sexos fueron T/P4, T/E2 y la sumatoria de los 3 metabolitos hormonales (Barja et al., 2006; Soto et al., 2004), y con el sirenio dugón (Dugong dugon) la relación T/E2 es mayor en machos que en hembras (Lanyon et al., 2005). Para el caso del venado cola blanca se obtuvieron eficiencias menores al $72.5 \%$ de la sumatoria de los 3 metabolitos a diferentes diluciones en la ENR, por lo que no resultan adecuados para asignar sexo en 
esta especie. Estos índices obtenidos del análisis de RIA fueron muy similares entre sexos en nuestro estudio, o tuvieron una alta variabilidad en sus valores, por lo que la distribución de valores de los índices entre ambos sexos se sobrepone y no se observa una clara distinción entre ellos.

Las eficiencias de los índices obtenidos con el método usado en este estudio (del 77.5-43.75\% en ENR y del 68.75-41.25\% en ER) fueron mayores que las conseguidas con el examen de la morfometría de pellets (un $42 \%$ en hembras, un $38 \%$ en machos, un $40.3 \%$ total) (Camargo-Sanabria, 2008). En contraste con el método usado en el presente estudio, la determinación del sexo basada en los rasgos morfométricos de los pellets se ve afectada por variaciones al tipo de alimentación, la humedad ambiental (Camargo-Sanabria y Mandujano, 2009), la ER o ENR (Maccracken y van Ballenberghe, 1987) y el grado de desarrollo corporal del individuo (Camargo-Sanabria y Mandujano, 2009; Maccracken y van Ballenberghe, 1987; Sánchez-Rojas et al., 2004).

En conclusión, se estimaron los niveles de metabolitos de progestágenos, estrógenos y andrógenos fecales satisfactoriamente, y el método utilizado permitió la determinación de los sexos con una mayor eficiencia que lo publicado con el método de morfometría de pellets, realizando la asignación de los mismos con base en el contenido diferencial de las hormonas esteroides sexuales. Este método para conocer la proporción de sexos en la población es posible aplicarlo en muestras en campo que sean frescas o con poco tiempo de exposición al ambiente.

\section{Agradecimientos}

Se agradece el financiamiento del Consejo Nacional de Ciencia y Tecnología (Conacyt) mediante el programa de Fondos Mixtos Conacyt y el Gobierno del Estado de Yucatán con el proyecto YUC-2006-C05-65725, así como la beca de Doctorado otorgada por el Conacyt núm. 47937. Al Campus de Ciencias Biológicas y Agropecuarias de la Universidad Autónoma de Yucatán por las facilidades otorgadas en el Laboratorio de Radioinmunoanálisis (RIA). Al zoológico «El Centenario», la UMA «Xmatkuil» y la UMA «San Fernando» por su colaboración para la ejecución de este proyecto. Así como a los 2 revisores anónimos por sus comentarios.

\section{Referencias}

Barja, I., Silván, G., Illera, J., Rosellini, S. y Piñeiro, A. (2006). La cuantificación de hormonas esteroides sexuales en heces de lobo ibérico (Canis lupus signatus): un método no invasivo de sexado como alternativa a los análisis moleculares. Oppidum, 2, 363-380.

Bedolla-Tovar, N., Ulloa-Aguirre, A., Landeros-Valpeña, J. y Pérez-Palacios, G. (1984). Análisis de datos y control de la calidad en el radioinmunoanálisis. I. Guía para la evaluación de resultados. Revista de Investigación Clínica, 36, 179-192.

Beringer, J., Hansen, L., Wilding, W., Fischer, J. y Sheriff, S. (1996). Factors affecting capture myopathy in white-tailed deer. Journal of Wildlife Management, 60, 373-380.

Camargo-Sanabria, A. (2008). Evaluación del conteo de grupos fecales y del análisis morfométrico de pellets como métodos de obtención de parámetros demográficos del venado cola blanca (Odocoileus virginianus mexicanus) en Puebla, México. Veracruz: Tesis de maestría. Instituto de Ecología.
Camargo-Sanabria, A. y Mandujano, S. (2009). Evaluación de la morfometría de pellets como método de categorización de sexo y edades en venado cola blanca (Odocoileus virginianus mexicanus) en Puebla, México. Revista Mexicana de Mastozoología, 13, 92-104.

Coltman, D. (2008). Molecular ecological approaches to studying the evolutionary impact of selective harvesting in wildlife. Molecular Ecology Journal Compilation, 1-15.

De Lucas, T., Zarco, Q. y Vásquez, P. (2008). El efecto macho como indicador de la actividad reproductiva en sistemas intensivos de apareamiento de ovinos. Veterinaria México, 39, 117-127.

Galen, S. R. (1979). New math in the lab. predictive value theory. Diagnostic Medicine, 2, 31-39.

Hamilton, R. A., Stanton, P. G., O’Donnell, L., Steele, V. R., Taggart, D. A. y Temple-Smith, D. (2000). Determination of seasonality in southern hairy-nosed wombats (Lasiorhinus latifrons) by analysis of fecal androgens. Biology of Reproduction, 63, 526-531.

Haulton, S., Porter, W. y Rudolph, B. (2001). Evaluating 4 methods to capture white-tailed deer. Wildlife Society Bulletin, 29, 255-264.

Herzog, K., Brockhan-Lüdemann, M., Kaske, M., Beindorff, N., Paul, V., Niemann, H., et al. (2010). Luteal blood flow is a more appropriate indicator for luteal function during the bovine estrous cycle tan luteal size. Theriogeno$\log y, 73,691-697$.

IAEA (International Atomic Energy Agency). (1984). Laboratory training manual on radioimmunoassay in animal reproduction. Viena: Technical Reports Series.

Lanyon, J., Smith, K. y Carrick, F. (2005). Reproductive steroids are detectable in faeces of dugongs. Australian Zoologist, 33, 247-250.

Latner, N. C., Rajamahendran, R. y Sivakumaran, K. (1994). Immunoreactive faecal progestins as indicator of reproductive status. Veterinary Record, 134, 474-475.

Lindsay, A. y Belant, J. (2008). A simple and improved PCR-based technique for white-tailed deer (Odocoileus virginianus) sex identification. Conservation Genetics, 9, 443-447.

Maccracken, J. y van Ballenberghe, V. (1987). Age- and sex-related differences in fecal pellet dimensions of moose. Journal of Wildlife Management, 51, 360-364.

Mandujano, S. y Gallina, S. (1993). Densidad del venado cola blanca basada en conteos en transectos en un bosque tropical de Jalisco. Acta Zoológica Mexicana (nueva serie), 56, 1-37.

Mandujano, S. y Gallina, S. (1996). Size and composition of white-tailed deer groups in a tropical dry forest in Mexico. Ethology, Ecology and Evolution, 8, 255-263.

Méndez-Villalobos, G., Jaramillo, G., Aragón, A., Ayala, M. y DomínguezVara, I. (2009). Función reproductiva de sementales ovinos importados de Nueva Zelanda durante su primera época reproductiva en México. Veterinaria México, 40, 123-131.

Milner, J., Nilsen, E. y Andreassen, H. (2007). Demographic side effects of selective hunting in ungulates and carnivores. Conservation Biology, 21, 36-47.

Montes, R. (1994). Validación de un radioinmunoanálisis en fase líquida para medir progesterona en plasma sanguíneo. Revista Biomédica, 5, 23-32.

Montes, R., Cervera, P. y Victoria, F. (1999). Diagnóstico de gestación en venadas cola blanca (Odocoileus virginianus) a través de la medición de metabolitos de progestinas fecales. En Asociación de zoológicos, criaderos y acuarios de la República Mexicana (Eds.) (pp. 25-28). Guadalajara: XVI Congreso de la Asociación de Zoológicos, Criaderos y Acuarios de la República Mexicana.

Palme, R. (2005). Measuring fecal steroids. Guidelines for practical application. Annals of the New York Academy of Sciences, 1046, 75-80.

Plotka, E., Seal, U., Schmoller, G., Karns, P. y Keenlyne, K. (1977). Reproductive steroids in the white-tailed deer (Odocoileus virginianus borealis). I. Seasonal changes in the female. Biology of Reproduction, 16, 340-343.

Plotka, E., Seal, U., Verme, L. y Ozoga, J. (1983). The adrenal gland in white-tailed deer: A significance source of progesterone. Journal of Wildlife Management, 47, 38-44.

Rabiee, A. R., Macmillan, K. L. y Schwarzenberg, F. (2001). The effect of level of feed intake on progesterone clearance rate by measuring faecal 
progesterone metabolites in grazing dairy cows. Animal Reproduction Science, 67, 205-214.

Sánchez-Rojas, G., Aguilar-Miguel, C. C. y Hernández-Cid, E. (2009). Estudio poblacional y uso de hábitat por el venado cola blanca (Odocoileus virginianus) en un bosque templado de la sierra de Pachuca, Hidalgo, México. Tropical Conservation Science, 2, 204-214.

Sánchez-Rojas, G., Gallina, S. y Equihua, M. (2004). Pellet morphometry as a tool to distinguish age and sex in the mule deer. Zoo Biology, 23, 139-146.

Soto, M. A., Salamé-Méndez, A., Ramírez-Pulido, J., Yáñez, L. y Armella, M.
A. (2004). Valoración de hormonas esteroides en heces de una pareja de

lobo mexicano (Canis lupus bailey) en cautiverio. Acta Zoológica Mexicana (nueva serie), 20, 187-196.

Tolleson, D., Randel, R., Stuth, J. y Neuendorff, D. (2005). Determination of sex and species in red and fallow deer by near infrared reflectance spectroscopy of the faeces. Small Ruminant Research, 57, 141-150.

Weber, M., García-Marmolejo, G. y Reyna-Hurtado, R. (2006). The tragedy of the commons: Wildlife management units in Southeastern Mexico. Wildlife Society Bulletin, 34, 1480-1488. 